\title{
HYPOVITAMINOSIS D AND MENOPAUSE: STILL AN ISSUE IN 2016?
}

\author{
Adina Ghemigian', Otilia Radu ${ }^{2}$, Ana Valea ${ }^{3}$, Nicoleta Dumitru ${ }^{4}$, Andra Buruiana ${ }^{4}$, Mara Carsote $^{1}$ \\ I"Carol Davila" University of Medicine and Pharmacy, \\ "C.I. Parhon" National Institute of Endocrinology, Bucharest \\ 2 "Carol Davila" University of Medicine and Pharmacy, Bucharest \\ 3"I. Hatieganu” University of Medicine and Pharmacy, Clinical County Hospital, Cluj-Napoca \\ 4 "C.I. Parhon" National Institute of Endocrinology, Bucharest
}

\begin{abstract}
Vitamin D in menopause is essential for skeleton and metabolic involvement. Our purpose is to introduce the level of evidence regarding menopause - related hypovitaminosis $D$ through a brief review of papers published on PubMed in 2016. The correlation between low vitamin D and risk of falls is a traditional observation. Lack of vitamin D remains one of the major contributors to osteoporotic fractures which have an annual incidence of 8.9 million worldwide. Yale FIT trial referring to menopausal cancer survivors after 12 months of aerobic physical exercise confirmed the bone loss under aromatase inhibitors opposite to tamoxifen is significant, a bone loss that is lower if vitamin $D$ ranges are low. Arzoxifene Generations Trial followed for 5 years menopausal women treated only with vitamin $D$ and calcium and found a fracture risk increase by $46 \%$ for each unit of lumbar T-score that decreases. A meta-analysis of 34 studies on 11,090 patients treated with ibandronate showed that hypovitaminosis $\mathrm{D}$ at baseline is a predictor of bone mineral density improvement only at lumbar spine. Another study revealed that $77 \%$ of menopausal females have 25 -hydoxyvitamin D (25$\mathrm{OH}$ D) less than $30 \mathrm{ng} / \mathrm{mL}$ while a negative correlation between 25-OH D and waist circumference was found knowing that android fat disposition in women with hipovitaminosis $D$ is a hallmark of metabolic syndrome. Overall, in 2016, PubMed published papers regarding vitamin D in menopause still reflect its deficiency and associated metabolic risk.
\end{abstract}

Keywords: menopause, hypovitaminosis D, vitamin D

\section{INTRODUCTION}

Vitamin D is a major element in many topics as bone, metabolic complications, some cancers, autoimmunity and many data have been provided in this matter for the last two decades.

\section{OBJECTIVE}

Our purpose is to introduce the level of evidence regarding menopause - related hypovitaminosis D.

\section{MATERIAL AND METHOD}

This is a brief narrative review over 2016 published English language papers from PubMed - indexed journals. The search based on words "vitamin D" and "menopause" found 35 papers and a selection of 15 has been done.

\section{GENERAL DATA}

Bone status is strongly influenced by vitamin D levels. The correlation between low vitamin D and risk of fall is a traditional observation nowadays. (1) JAMA introduced large debates related to criteria of adequate vitamin D levels in patients enrolled in different studies. (2-5) The skeleton state is tidily connected to vitamin D values; if hypovitaminosis D is avoided, better response to anti-osteoporotic drugs is seen and lower bone damage secondary to different medication as anti-cancer medication is detected. (6) Yale FIT trial referring to menopausal cancer survivors after 12 months of aerobic physical exercise confirmed the bone loss under aromatase inhibitors opposite to tamoxifen is significant, a bone loss that is lower if vitamin D ranges are between 20 and $29 \mathrm{ng} / \mathrm{mL}$. (6) Large cohort of 974 women form Arzoxifene Generations 
Trial followed for 5 years, menopausal women treated only with vitamin $\mathrm{D}$ and calcium and found a fracture risk increase by $46 \%$ for each unit of lumbar T-score that decreases. (7) A meta-analysis from PlosOne, gathering 34 studies on 11090 patients treated with ibandronate showed that hypovitaminosis D at baseline is a predictor of bone mineral density improvement only at lumbar spine while overall, best predictors of good response were a duration of therapy between one to five years, low T-score, advanced age, etc. (8) As answer to the question how afraid to be of aggressive vitamin D supplementation the evidence showed that bone and muscle are not altered after one year. (9) Lack of vitamin D remains one of the major contributors to osteoporotic fractures which have an annual incidence of 8.9 million. (10)

\section{Metabolic connections are established with hypovitaminosis D}

A large Chinese study found that 25-hydroxyvitamin D (25-OH D) and sex hormone binding globulin is inversely correlated with non-alcoholic fatty liver disease, but the exact cause-effect relationship remains unknown. (11) A cross-sectional study from Nutrition revealed that $77 \%$ of menopausal females have $25-\mathrm{OH}$ D less than $30 \mathrm{ng} / \mathrm{mL}$. (12)
There is a negative correlation between $25-\mathrm{OH} \mathrm{D}$ and waist circumference while android fat disposition found in women with hipovitaminosis D (as confirmed by DXA assessment) is a hallmark of metabolic syndrome. (12) On the other hand, a study on 218 obese women aged between 50 and 75 years associating vitamin $\mathrm{D}$ deficiency who lost weight during a year pointed that vitamin D supplementation involves a larger reduction on sex hormones when compare to patients without vitamin D repletion. (13) A high prevalence of vitamin D deficiency among menopausal females with type 2 diabetes mellitus up to $89 \%$ was found in one study without a correlation between 25-OH D and glycated haemoglobin, disease duration, etc. (14)

\section{Various domains also involve vitamin D}

For instance, Spanish consensus regarding people who take anti-psychotic medication and have associated hyperprolactinemia indicates initially testing vitamin D in every patient (15).

\section{CONCLUSION}

Overall, in 2016, PubMed published papers regarding vitamin $\mathrm{D}$ in menopause still reflect its deficiency and associated metabolic risk.

\section{REFERENCES}

1. Palacios S. Association between vitamin $D$ and falls in young postmenopausal women. Menopause. 2016 Mar; 23(3):239-40.

2. Karnchanasorn R., Ou H.Y., Chiu K.C. Proposed Guidelines for Future Vitamin D Studies. JAMA Intern Med. 2016 Feb; 176(2):280-1.

3. Hansen K.E., Johnson M.G. Proposed Guidelines for Future Vitamin D Studies - Reply. JAMA Intern Med. 2016 Feb; 176(2):281-2.

4. Grant W.B., Mascitelli L., Goldstein M.R. Proposed Guidelines for Future Vitamin D Studies. JAMA Intern Med. 2016 Feb; 176(2):279-80.

5. Tufan A.N., Tufan F. Proposed Guidelines for Future Vitamin D Studies. JAMA Intern Med. 2016 Feb; 176(2):280.

6. Knobf M.T., Jeon S., Smith B., et al. Effect of a randomized controlled exercise trial on bone outcomes: influence of adjuvant endocrine therapy. Breast Cancer Res Treat. 2016 Feb; 155(3):491-500.

7. Arantes H.P., Gimeno S.G., Chiang A.Y., et al. Incidence of vertebral fractures in calcium and vitamin D-supplemented postmenopausal Brazilian women with osteopenia or osteoporosis: data from Arzoxifene Generations Trial. Arch Endocrinol Metab. 2016 Feb; 60(1):54-9.

8. Ma Z., Li Y,. Zhou M., et al. Predictors of Ibandronate Efficacy for the Management of Osteoporosis: A Meta-Regression Analysis. PLoS One. 2016 Mar 1; 11(3):e0150203. doi: 10.1371/journal.pone.0150203.
9. Harvey N.C., Cooper C. High-dose vitamin D supplementation does not alter bone mass or muscle function over 1 year in postmenopausal women. Evid Based Med. 2016 Feb; 21(1):30.

10. Pisani P., Renna M.D., Conversano F., et al. Major osteoporotic fragility fractures: Risk factor updates and societal impact. World J Orthop. 2016 Mar 18;7(3):171-81.

11. Wang N., Zhai H., Zhu C., et al. Combined Association of Vitamin D and Sex Hormone Binding Globulin With Nonalcoholic Fatty Liver Disease in Men and Postmenopausal Women: A Cross-Sectional Study. Medicine (Baltimore). 2016 Jan; 95(4):e2621.

12. Andreozzi P., Verrusio W., Viscogliosi G., et al. Relationship between vitamin $D$ and body fat distribution evaluated by DXA in postmenopausal women. Nutrition. 2016 Jun;32(6):687-92.

13. Mason C, De Dieu Tapsoba J., et al. Effects of vitamin D supplementation during weight loss on sex hormones in postmenopausal women. Menopause. 2016 Jun; 23(6):645-52.

14. Raška I. Jr, Rašková M., Zikán V., Škrha J. High Prevalence of Hypovitaminosis D in Postmenopausal Women with Type 2 Diabetes Mellitus. Prague Med Rep. 2016; 117(1):5-17.

15. Montejo Á.L., Arango C., Bernardo M., et al. Spanish consensus on the risks and detection of antipsychotic drug-related hyperprolactinaemia. Rev Psiquiatr Salud Ment. 2016 Jul-Sep;9(3):158-73. 